\title{
Horse flies (Diptera: Tabanidae) collected in Central African Republic, Gabon and Liberia with comments on their updated distribution
}

\author{
Jan Ježek, Jan Votýpka, Jana Brzoňová \& Jozef Oboňa
}

\begin{abstract}
Horse flies (Diptera: Tabanidae) collected in Central African Republic, Gabon and Liberia with comments on their updated distribution. - Acta Mus. Siles. Sci. Natur., 68: 263-274, 2019.

Abstract: A zoogeographical review summarized the current distribution of 28 morphologically determined horse fly species recently collected in three Western Africa countries. In Malaise trap collections, the family Tabanidae was represented by three subfamilies, Pangoniinae, Chrysopsinae and Tabaninae (tribes Philolichini, Chrysopsini, Tabanini and Haematopotini), and six genera: Philoliche Wiedemann, 1828 (1 species), Chrysops Meigen, 1803 (3 specis), Ancala Enderlein, 1922 (2 species), Euancala Enderlein, 1922 (1 species), Tabanus Linnaeus, 1758 (13 species) and Haematopota Meigen, 1803 (8 species). Information contained in the principal publication The Catalogue of the Diptera of the Afrotropical Region 1980 is complemented by newly published studies on horse flies in Sub-Saharan Africa.
\end{abstract}

Key words: Taxonomy, faunistics, horse fly, Tabanids, distribution, CAR, Gabon, Liberia, Afrotropical Region

\section{Introduction}

In the Afrotropical Region, the family Tabanidae is represented by approximately 800 species in 35 genera (Chainey 2017). Many horse fly species have medical and veterinary importance as vectors of human and livestock pathogens causing various diseases (e.g., asanaplasmosis, anthrax, animal trypanosomiasis, bovine viral leukosis, filarial worms, tularaemia, hog cholera, vesicular stomatitis). Traditional taxonomic tools based on morphological diagnostic characters and descriptions are limited in contrast to modern molecular approaches. However, only the combination of both these methods can provide relevant information and continuity with previous publications. The horse fly fauna of tropical Africa is still poorly known and in some geographical areas was not studied for decades. This study summarizes the results of tabanid collections made in three West African countries, the Central African Republic (CAR), Gabon and Liberia, from where only very sparse information was previously available.

\section{Material and methods}

The abundant horse fly samples were collected by J. Votýpka and J. Brzoňová by using Malaise traps (Figs 1-4) and sweep netting in three Western Africa countries: CAR - September 2012, Gabon - May to July 2014, and Liberia - September/October 2016 (localities and coordinates are mentioned in the text). All specimens conserved in ethanol (see Votýpka et al., 2019) were identified by the first author by using Carl Zeiss stereomicroscope (Citoval 2). However, the content of this faunistic study represents only 171 (170 females and one male) pined specimens arranged into a small comparative dry collection deposited in the National Museum in Prague (NMPC).

The morphological identification was made mainly based on the historical works of Oldroyd, with crucial descriptions, figures and photographs (monographs of African horse flies with keys - 1952, 1954, 1957). The nomenclature of the family Tabanidae was mainly used sensu Moucha (1976), and partially sensu Chainey \& Oldroyd (1980). The molecular part of the study including DNA barcoding of morphologically determined horse flies, their intra- and interspecific divergences and pathogen occurrence were published by Votýpka et al. (2019). 

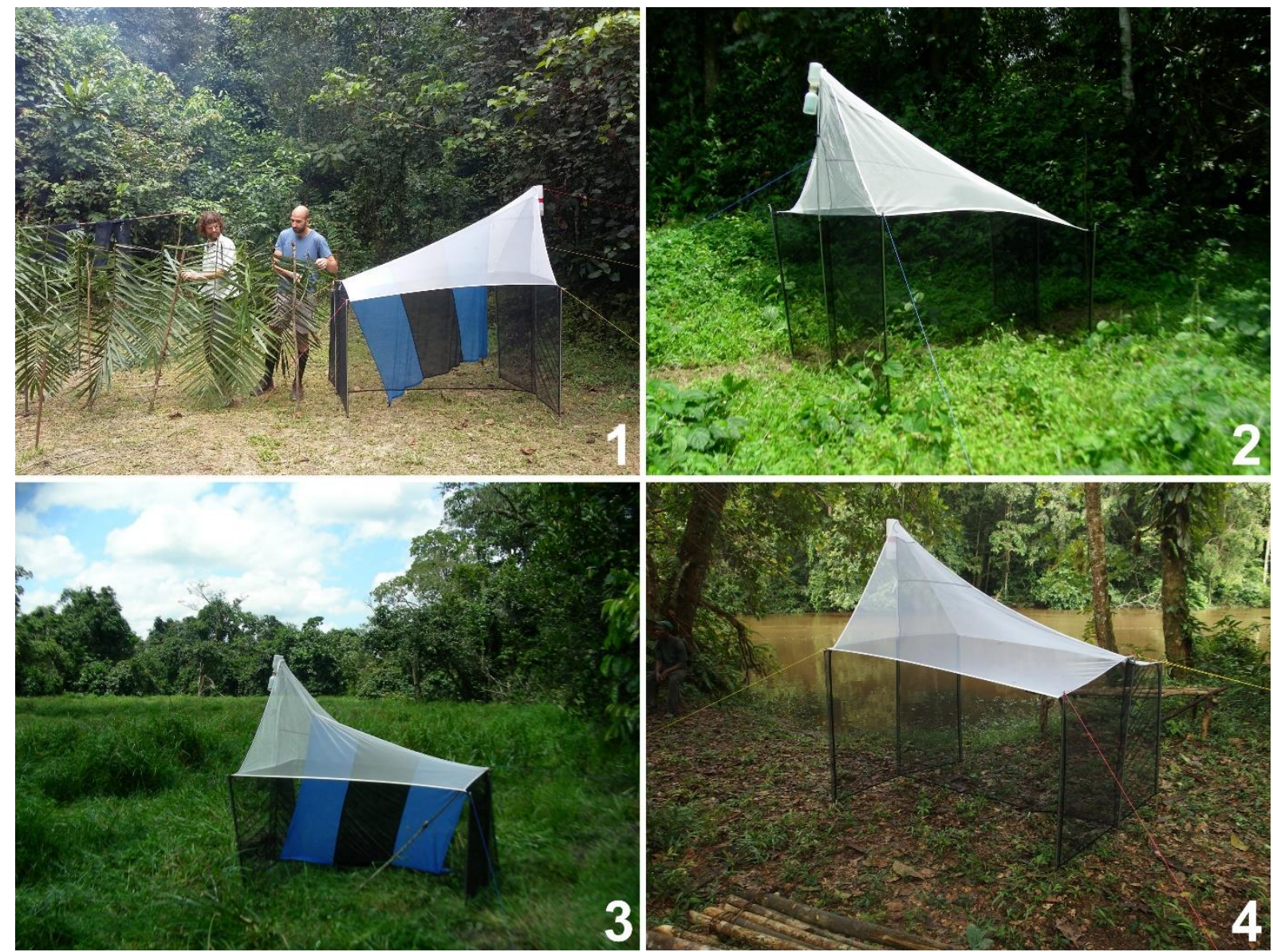

Figs 1-4: 1 - A tent-like Malaise trap with a black and dark-blue striped central wall localized in a clearing near the Viro camp located in primary dense forest near the border of Sapo NP, Liberia. The trap is complemented by a wall (made from palm leaves) that directs flying insects into the central part. 2 - A Malaise trap localized in a clearing of the dense forest, Dzanga-Sangha Protected Areas (DSPA), the Central African Republic (CAR). 3 - A Malaise trap with a black and dark-blue striped central wall localized in a huge clearing (a "bai") of the primary forest, Dzanga-Sangha Protected Areas (DSPA), the Central African Republic (CAR). 4 - A Malaise trap, surrounding by a secondary forest, is localized on the Sinoe River bank forming the border of Sapo NP, Liberia (photos made by David Modrý and Jan Votýpka).

\section{Results}

\section{TABANIDAE PANGONIINAE PHILOLICHINI}

Philoliche Wiedemann, 1828: Aussereur. Zweifl. Ins., 1: 95.

Subpangonia Surcouf, 1908: Bull. Mus. Hist. Nat. Paris, 14: 283.

1. Philoliche (Subpangonia) gravoti Surcouf, 1908: Bull. Mus. Hist. Nat. Paris, 14: 283.

Material examined: Liberia, VIRO camp, Sapo NP, $5^{\circ} 18^{\prime} 39.89^{\prime \prime} \mathrm{N}, 8^{\circ} 46^{\prime} 53.07^{\prime \prime} \mathrm{W}, 130$ a.s.l., dense forest, 4 우, ix./x. 2016, MT, J. Votýpka leg. Liberia, Jelay x VIRO, 5²0'14.92"N, 847'58.44"W, 125 m a.s.l., river, forest, 2 우, ix./x. 2016, MT, J. Votýpka leg. (dry, pined, NMPC).

Chronology of selected important zoogeographical views. Oldroyd (1957): former Belgian Congo, French Equatorial Africa, the Camerouns, French Guinea (adapted); Nigeria. Moucha (1976): former Equat. Africa (adapted). Chainey \& Oldroyd (1980): Gabon, Ghana, Guinea, Nigeria, former Cameroun, Congo and Zaire (adapted). Morita (2008): Gabon.

Distribution (only verified precise location from the last period): Cameroon, Congo Republic, DRC, Gabon, Ghana, Guinea, Nigeria. Published as new for Liberia by Votýpka et al. (2019). 


\section{CHRYSOPSINAE CHRYSOPSINI}

Chrysops Meigen, 1803: Illig. Mag., 2: 267.

2. Chrysops dimidiatus Wulp, 1885: Notes Leyden Mus., 7: 80.

Material examined: CAR, Dzanga-Sangha NP, Bayanga, Bai Hokou, camp, 1 ๆ, 25-30.ix.2012, SW, J. Votýpka leg. (dry, pined, NMPC).

Chronology of selected important zoogeographical views. Oldroyd (1957): S.W. Africa; former Congo, British and French Cameroons, Guinea (adapted), Benin, Nigeria. Leclercq (1961): former Congo (adapted). Moucha (1976): Ethiopian Region. Chainey \& Oldroyd (1980): former Cameroun, Congo and Zaire (adapted), Angola, Central African Republic, Ghana, Guinea, Nigeria. Gouteux et al. (1989), Noireau \& Gouteux (1989), Noireau et al. (1990 a,b), Noireau et al. (1991), Caubère et al. (1990), Caubere \& Noireau (1991): Congo Republic and DRC (adapted). Pinder (1991), Wahl et al. (1995), Kelly- Mavoungou et al. (2012): Gabon. Dias (1994): Angola. Dias (1996), Hope et al. (2012): CAR. Chippaux et al. (2000), Demanou et al. (2001): Cameroon. Cheke et al. (2003): Bioko Island - Equatorial Guinea Republic. Iboh et al. (2012): Nigeria.

Distribution (only verified precise location from the last period): Angola, Benin, Bioko Island - Equatorial Guinea Republic, Cameroon, CAR, Congo Republic, DRC, Gabon, Ghana, Guinea, Nigeria.

3. Chrysops longicornis Macquart, 1838: Dipt. exot., 1: 156.

Material examined: CAR, Dzanga-Sangha NP, Bayanga, Bai Hokou, camp, 1 ㅇ, 26.ix.2012, SW, J. Votýpka leg.; same, Bai-Gubunga, 5 웅, 1 §, 28.ix.2012, MT, J. Votýpka leg.; Gabon, Loango NP, Ndouani, 4 우우, 11.15.vi.2014, hand collecting, J. Brzoňová leg.; same, 2 우우, 12.vi.-9.vii.2014, NZI tsetse trap, J. Brzoňová leg. (dry, pined, NMPC). Liberia, VIRO camp, Sapo NP, $5^{\circ} 18^{\prime} 39.89^{\prime \prime} \mathrm{N}, 8^{\circ} 46^{\prime} 53.07^{\prime \prime} \mathrm{W}, 130$ a.s.l., dense forest, 1 , ix./x. 2016, MT, J. Votýpka leg., (dry, pined, NMPC).

Chronology of selected important zoogeographical views. Oldroyd (1957): Ethiopian Region, Cape Province Natal, Zanzibar, Pemba Island (Zanzibar Archipelago), former British Cameroons (adapted). Leclercq (1961): former Congo (adapted). Leclercq (1965): Liberia. Moucha (1976): Ethiop. Region. Chainey \& Oldroyd (1980): Senegal; widespread Afrotrop. reg. (incl. Pemba and Zanzibar), Sierra Leone. Goodwin (1982, 1985): Mali. Raymond et al. (1984): Senegal. Dias (1987, 1994): Angola. Inaoka et al. (1988), Hayakawa et al. (1989), Itina et al. (2013): Nigeria. Chainey \& Cheke (1994): Togo. Taylor \& Chainey (1994), Acapovi et al. (2001): Ivory Coast. Dias (1996): CAR. Sasaki \& Nishida (1999), Sasaki (2005): Tanzania. Schacht (2000): Gambia. Dia et al. (2004), Koné et al. (2011): Burkina Faso. Esterhuizen (2006): Natal Province, South Africa.

Distribution (only verified precise location from the last period): Angola, Burkina Faso, Cmeroon, CAR, Congo Republic, DRC, Gambia, Ivory Coast, Liberia, Mali, Natal Province - South Africa, Nigeria, Pemba Island, Senegal, Sierra Leone Republic, Tanzania, Togo. Published as new for Gabon by Votýpka et al. (2019).

4. Chrysops silaceus Austen, 1907: Ann. Mag. nat. Hist. 20(7): 509.

Material examined: CAR, Dzanga-Sangha NP, Bai Hokou, camp, 8 q + , 25.-30.ix.2012, SW, J. Votýpka leg.; same, 1 q, 13.ix.2014, MT, A. Mihalica and D'. Amico leg. (dry, pined, NMPC).

Chronology of selected important zoogeographical views. Oldroyd (1957): S. and N. Nigeria, Sudan, former Gold Coast, Cameroons and Belgian Congo (adapted). Moucha (1976): Ethiop. Region. Chainey \& Oldroyd (1980): Angola, Central African Republic, Fernando Póo, Ghana, Nigeria, Sudan, former Congo, Cameroun and Zaire (adapted). Inaoka et al. (1988), Hayakawa et al. (1989), Iboh et al. (2012), Itina et al. (2013): Nigeria. Gouteux et al. (1989), Noireau \& Gouteux (1989), Caubère et al. (1990), Caubere \& Noireau (1991), Noireau et al. (1990 a,b), Noireau et al. (1991): Congo Republic, DRC (adapted). Pinder (1991), Wahl et al. (1995), Mavoungou et al. (2012): Gabon. Dias (1994): Angola. Dias (1996), Kelly-Hope et al. (2012): CAR (adapted). Chippaux et al. (2000), Demanou et al. (2001), Wanji et al. (2002): Cameroon (adapted). Cheke et al. (2003): Bioko Island - Equatorial Guinea Republic.

Distribution (only verified precise location from the last period): Angola, Bioko Island and Fernando Póo Island - Equatorial Guinea Republic, Cameroon, CAR, Congo Republic, DRC, Gabon, Ghana, Nigeria, Sudan. 


\section{TABANINAE. TABANINI}

Ancala Enderlein, 1922: Mitt. zool. Mus. Berlin, 10: 346.

5. Ancala fasciata (Fabricius, 1775): Syst. Ent., p. 789.

Material examined: Gabon, Loango NP, Petit Loango, 2 우, 10.-17.v.2014, 17.-19.vi.2014, MT, J. Brzoňová leg. (dry, pined, NMPC).

Chronology of selected important zoogeographical views. Oldroyd (1954): West African sub-region of Chapin. Leclercq (1965): former Congo (adapted). Moucha (1976): W. Africa. Chainey \& Oldroyd (1980): Sierra Leone; widespread throughout West African subregion and surrounding savanas. Dusbábek et al. (1980): Uganda. Goodwin (1982): Mali. Dias (1987, 1992): Guinea Bissau (adapted). Hayakawa et al. (1989): Nigeria. Amsler et al. (1994), Dia et al. (2004), Koné et al. (2011): Burkina Faso. Chainey \& Cheke (1994): Togo. Dias (1994): Angola. Taylor \& Chainey (1994), Acapovi et al. (2001): Ivory Coast. Dias (1996): CAR. Mavoungou et al. (2012): Gabon.

Distribution (only verified precise location from the last period): Angola, Burkina Faso, CAR, Congo Republic, DRC, Gabon, Guinea Bissau, Ivory Coast, Mali, Nigeria, Sierra Leone Republic, Togo, Uganda.

6. Ancala fasciata f. mixta (Surcouf, 1914): Rev. Zool. Afr., 3: 472.

Material examined: CAR, Dzanga-Sangha NP, Bai Hokou, 1 q, 13.ix.2014, MT, A. Mihalica and D'. Amico leg. (dry, pined, NMPC).

Chronology of selected important zoogeographical views. Oldroyd (1954): West African sub-region of Chapin. Moucha (1976): W. Africa. Chainey \& Oldroyd (1980): former Zaire (adapted).

Distribution (only verified precise location from the last period): DRC. Published as new for CAR by Votýpka et al. (2019), however, some sibling species in the case of Ancala fasciata are discussed here.

Euancala Enderlein, 1922: Mitt. zool. Mus. Berlin, 10: 346.

7. Euancala irrorata (Surcouf, 1909): Bull. Mus. Hist. Nat. Paris, 15: 355.

Material examined: Gabon, Loango NP, Petit Loango, 1 o, 10.-17.v.2014, MT, J. Brzoňová leg. (dry, pined, NMPC).

Chronology of selected important zoogeographical views. Oldroyd (1954): Sierra Leone to Uganda, former Gaboon and Belgian Congo (adapted). Moucha (1976): S. Leone to Uganda. Chainey \& Oldroyd (1980): Gabon; Angola, from Sierra Leone to Uganda \& former Zaire (adapted). Dias (1994): Angola. Dias (1996): CAR.

Distribution (only verified precise location from the last period): Angola, CAR, Congo Republic, DRC, Gabon, Sierra Leone Republic, Uganda.

Tabanus Linnaeus, 1758: Syst. Nat., ed. 10: 601.

8. Tabanus argenteus Surcouf, 1907: Bull. Mus. Hist. Nat. Paris, 13: 263.

Material examined: Liberia, VIRO camp, Sapo NP, $5^{\circ} 18^{\prime} 39.89^{\prime \prime} \mathrm{N}, 8^{\circ} 46^{\prime} 53.07^{\prime \prime} \mathrm{W}, 130$ a.s.l., dense forest, 4 우, ix./x. 2016, MT, J. Votýpka leg. Same, Jelay x VIRO, $5^{\circ} 20^{\prime} 14.92^{\prime \prime N}, 8^{\circ} 47^{\prime} 58.44 " \mathrm{~W}, 125 \mathrm{~m}$ a.s.l., river, forest, 1 †, ix./x. 2016, MT, J. Votýpka leg., (dry, pined, NMPC).

Chronology of selected important zoogeographical views. Oldroyd (1954): from Sierra Leone to the former Belgian Congo (adapted). Leclercq (1965): Liberia. Moucha (1976): former Congo (adapted). Chainey \& Oldroyd (1980): Gabon, from Guinea and Sierra Leone to Central African Republic and former Zaire (adapted). Inaoka et al. (1988): Nigeria. Dias (1994): Angola. Taylor \& Chainey (1994): Ivory Coast. Dias (1996): CAR. Cheke et al. (2003): Bioko Island - Equatorial Guinea Republic.

Distribution (only verified precise location from the last period): Angola, Bioko Island - Equatorial Guinea Republic, CAR, Congo Republic, DRC, Gabon, Guinea, Ivory Coast, Liberia, Nigeria, Sierra Leone Republic.

9. Tabanus boueti Surcouf, 1907: Bull. Mus. Hist. Nat. Paris, 13: 333.

Material examined: Liberia, VIRO camp, Sapo NP, $5^{\circ} 18^{\prime} 39.89^{\prime \prime} \mathrm{N}, 8^{\circ} 46^{\prime} 53.07^{\prime \prime} \mathrm{W}, 130$ a.s.l., dense forest, 6 우, ix./x. 2016, MT, J. Votýpka leg., (dry, pined, NMPC).

Chronology of selected important zoogeographical views. Oldroyd (1954): Guinean Forest area, from former 
Gold Coast through the Ivory Coast to former Dahomey and Belgian Congo (adapted). Moucha (1976): W. and C. Africa. Chainey \& Oldroyd (1980): Ivory Coast, southern W. Afr. from Guinea and Liberia to former Zaire (adapted). Taylor \& Chainey (1994), Acapovi et al. (2001): Ivory Coast.

Distribution (only verified precise location from the last period): Benin, Congo Republic, DRC, Ghana, Guinea, Ivory Coast, Liberia.

10. Tabanus fraternus Macquart, 1846: Dipt. exot. Suppl., 1: 31.

Material examined: CAR, Dzanga-Sangha NP, Bai Hokou, 2 qq, 22.ix.2014, MT, A. Mihalica and D'. Amico leg.; same, Bayanga, Bai Hokou, camp, 1 , 26.ix.2012, MT, J. Votýpka leg.; same, Bai-Gubunga, 3 우, 28.ix.2012, MT, J. Votýpka leg. (dry, pined, NMPC).

Chronology of selected important zoogeographical views. Oldroyd (1954): from the former Cape Colony to Kenya and to former Belgian Congo, former Abyssinia and Senegambia, Eritraea, Sudan, Tanganyika (adapted). Moucha (1976): Ethiopian Region. Chainey \& Oldroyd (1980): South Africa; S.-E. Afr. from Kenya and Burundi to sthn Afr. (incl. Angola), ? W. Afr. Wiesenhütter (1980): Tanzania. Dias (1994): Angola.

Distribution (only verified precise location from the last period): Angola, Burundi, Congo Republic, DRC, Eritraea, Ethiopia, Gambia, JAR, Kenya, Senegal, Sudan, Tanzania. Published as new for CAR by Votýpka et al. (2019).

11. Tabanus ianthinus Surcouf, 1907: Bull. Mus. Hist. Nat. Paris, 13: 212.

Material examined: Liberia, VIRO camp, Sapo NP, 5¹8'39.89"N, $8^{\circ} 46^{\prime} 53.07^{\prime \prime} \mathrm{W}, 130$ a.s.1., dense forest, 10 우, ix./x. 2016, MT, J. Votýpka leg. Same, Jelay x VIRO, 5²0'14.92"N, 847'58.44"W, $125 \mathrm{~m}$ a.s.1., river, forest, 16 우, ix./x. 2016, MT, J. Votýpka leg., (dry, pined, NMPC).

Chronology of selected important zoogeographical views. Oldroyd (1954): former Belgian Congo, French Congo, Cameroun Français; Angola, Sierra Leone and Senegal (adapted). Moucha (1976): Angola, former Congo and Cameroons (adapted). Chainey \& Oldroyd (1980): Angola, Senegal, Sierra Leone, former Congo and Zaire (adapted). Raymond et al. (1984): Senegal. Dias (1987, 1994): Angola. Dias (1996): CAR.

Distribution (only verified precise location from the last period): Angola, Cameroon, CAR, Congo Republic, DRC, Senegal, Sierra Leone Republic. Published as new for Liberia by Votýpka et al. (2019).

12. Tabanus obscurehirtus Ricardo, 1908: Ann. Mag. nat. Hist., (8)1: 274.

Material examined: CAR, Dzanga-Sangha NP, Bai-Gubunga, 3 q + , 28.ix.2012, MT, J. Votýpka leg.; same, Bayanga, Bai Hokou, camp, 1 †, 26.ix.2012, MT, J. Votýpka leg.; same, Mongambe, camp, 2 q , , 22.ix.2012, SW, J. Votýpka leg.; same, Bai Hokou, 1 q, 20.ix.2014, MT, A. Mihalica and D'. Amico leg. (dry, pined, NMPC).

Chronology of selected important zoogeographical views. Oldroyd (1954): Guinean Forest area, Liberia, S. Nigeria, Southern Cameroons; former French and Belgian Congo, French Equatorial Africa (adapted). Moucha (1976): Equat. Africa. Chainey \& Oldroyd (1980): southern W. Afr. from Liberia to Nigeria, Angola, Central African Republic, former Congo and Zaire (adapted). Dias (1987, 1994): Angola. Dias (1996): CAR. Mavoungou et al. (2012): Gabon.

Distribution (only verified precise location from the last period): Angola, Cameroon, CAR, Congo Republic, DRC, Gabon, Guinea, Liberia, Nigeria.

13. Tabanus par Walker, 1854: List Dipt. Brit. Mus. 5, Suppl. 1: 235.

Material examined: CAR, Dzanga-Sangha NP, Bai-Gubunga, 3 q, , 28.ix.2012, MT, J. Votýpka leg.; Gabon, Loango NP, Petit Loango, 2 q + , 10.-17.v.2014, 22.-25.v.2014, MT, J. Brzoňová leg. (dry, pined, NMPC).

Chronology of selected important zoogeographical views. Oldroyd (1954): from the Gambia to Natal, Uganda, former Belgian Congo (adapted). Leclercq (1961): former Congo (adapted). Leclercq (1965): Angola. Moucha (1976): Gambia to Natal. Chainey \& Oldroyd (1980): South Africa; throughout Afrotrop. Africa but mainly outside Congo basin. Dusbábek et al. (1980): Uganda. Goodwin (1982, 1985), Djiteye et al. (1998): Mali. Raymond et al. (1984): Senegal. Amsler et al. (1994), Dia et al. (2004): Burkina Faso. Dias (1985): Guinea Bissau. Dias (1994): Angola. Dias (1996): CAR. Acapovi et al. (2001): Ivory Coast. Ahmed et al. (2005): Nigeria. Esterhuizen (2006): Natal Province, South Africa. Mavoungou et al. (2012), Bitome Essono et al. (2015): Gabon.

Distribution (only verified precise location from the last period): Angola, Burkina Faso, CAR, Congo Republic, DRC, Gabon, Gambia, Guinea Bissau, Ivory Coast, Mali, Natal Province - South Africa, Nigeria, Senegal, Uganda. 
14. Tabanus rufipes Palisot de Beauvois, 1806: P1. Diptères I, fig. 3

Material examined: Liberia, VIRO camp, Sapo NP, $5^{\circ} 18^{\prime} 39.89^{\prime \prime} \mathrm{N}, 8^{\circ} 46^{\prime} 53.07^{\prime \prime} \mathrm{W}, 130$ a.s.l., dense forest, 9 우우, 1 M, ix./x. 2016, MT, J. Votýpka leg.; same, Jelay x VIRO, 5²0'14.92"N, 847'58.44"W, 125 m a.s.1., river, forest, 11 우, ix./x. 2016, MT, J. Votýpka leg., (dry, pined, NMPC).

Chronology of selected important zoogeographical views. Oldroyd (1954): from the former Gold Coast (adapted) through the Katanga to Nyasaland and Angola, Sudan (as ruficrus). Leclercq (1965): Liberia and Guinea (as ruficrus). Moucha (1976): former Congo (adapted), Uganda (as ruficrus). Chainey \& Oldroyd (1980): Nigeria, through southern W. Afr. to Congo basin, Sudan, Uganda, Malawi and Angola (as rufipes), Gabon (as deyrollei and dilutius), Zambia (as grandissimus). Dias (1994): Angola (as ruficrus). Taylor \& Chainey (1994): Ivory Coast (as ruficrus).

Distribution (only verified precise location from the last period): Angola, Congo Republic, DRC, Gabon, Ghana, Guinea, Ivory Coast, Liberia, Malawi, Nigeria, Sudan, Uganda, Zambia.

\section{Tabanus secedens Walker, 1854: List Dipt. Brit. Mus. 5, Suppl. 1: 224.}

Material examined: CAR, Dzanga-Sangha NP, Bayanga, Bai Hokou, camp, 4 q, , 26.ix.2012, MT, J. Votýpka leg.; Gabon, Loango NP, Petit Loango, 2 o o, 10.-17.v.2014, 1 F 22.-25.v.2014, MT, J. Brzoňová leg. (dry, pined, NMPC). Liberia, VIRO camp, Sapo NP, 5¹8'39.89"N, 846'53.07"W, 130 a.s.l., dense forest, 4 우, ix./x. 2016, MT, J. Votýpka leg.; same, Jelay x VIRO, 5²0'14.92"N, 847'58.44"W, $125 \mathrm{~m}$ a.s.1., river, forest, 5 우, ix./x. 2016, MT, J. Votýpka leg., (dry, pined, NMPC).

Chronology of selected important zoogeographical views. Oldroyd (1954): from Sierra Leone to Uganda, Nigeria and Sudan, the former Belgian Congo and Cameroons (adapted). Leclercq (1961): former Congo (adapted). Leclercq (1965): Liberia. Moucha (1976): S. Leone to former Congo (adapted). Chainey \& Oldroyd (1980): throughout West African subregion from Sierra Leone to Uganda, also into Sudan, Malawi and Angola. Dias (1987, 1994): Angola. Hayakawa et al. (1989, Itina et al. (2013), Ahmed et al. (2005): Nigeria (second author as seledens). Taylor \& Chainey (1994): Ivory Coast. Dias (1996): CAR. Mavoungou et al. (2012): Gabon.

Distribution (only verified precise location from the last period): Angola, Cameroon, CAR, Congo Republic, DRC, Gabon, Ivory Coast, Liberia, Malawi, Nigeria, Sierra Leone Republic, Sudan, Uganda.

16. Tabanus secedens f. regnaulti Surcouf, 1912: Bull. Soc. ent. France, p. 183.

Material examined: Gabon, Loango NP, Petit Loango, 4 q $q$, 10.-17.v.2014, 1 q, 22.-25.v.2014, all MT, J. Brzoňová leg. (dry, pined, NMPC). Liberia, VIRO camp, Sapo NP, 5¹8'39.89"N, 8 46'53.07"W, 130 a.s.1., dense forest, 2 + $q_{\text {, }}$, ix./x. 2016, MT, J. Votýpka leg.; same, Jelay x VIRO, 5²0'14.92"N, 847'58.44"W, 125 m a.s.1., river, forest, 5 우, ix./x. 2016, MT, J. Votýpka leg., (dry, pined, NMPC).

Chronology of selected important zoogeographical views. Oldroyd (1954): Sierra Leone, former Gold Coast, Belgian Congo and British Cameroons, as syn. of secedens, however, bona species? (adapted). Moucha (1976): S. Leone to former Congo, as syn. of secedens (adapted). Chainey \& Oldroyd (1980): former Congo, as syn. of secedens. Dias (1994): Angola. Taylor \& Chainey (1994): Ivory Coast.

Distribution (only verified precise location from the last period): Angola, Cameroon, Congo Republic, DRC, Ghana, Ivory Coast, Sierra Leone Republic. Published as new for Gabon and Liberia by Votýpka et al. (2019). A genetic heterogenity of Tabanus secedens complex was discussed in the mentioned paper.

17. Tabanus taeniola Palisot de Beauvois, 1807: Ins. rec. Afr. Amer., 1: 56.

Material examined: CAR, Dzanga-Sangha NP, Bai-Gubunga, 3 qq, 28.ix.2012, MT, J. Votýpka leg.; Gabon, Loango NP, Petit Loango, 3 우, 10.-17.v.2014, 1 + , 22.-25.v.2014, 1 , 17.-19.vi.2014, MT; same, Ndouani, 1 , 11.-15.vi.2014, hand collecting, all J. Brzoňová leg. (dry, pined, NMPC).

Chronology of selected important zoogeographical views. Oldroyd (1954): Unusual distribution, see the map 26, p. 282, mainly old literary data; e.g. Senegal, Angola, Eritraea, Somalilands, Cape Colony, Rhodesias, former Abyssinia, Belgian Congo and French Equatorial Africa (excessively adapted). Steyskal \& El-Bialy (1967), Morsy \& Habib (2001), Mohamed (2002): Egypt. Moucha (1976) Ethiopian Region. Chainey \& Oldroyd (1980): Nigeria; almost entire Afrotropical Region (incl. Aldabra and Madagascar) but mainly riverine in Congo basin. Dusbábek et al. (1980): Uganda. Karim (1980), Mahmoud \& Osman (1979): Sudan.. Wiesenhütter (1980), Sasaki \& Nishida (1999), Sasaki (2005): Tanzania. Goodwin (1982, 1985), Djiteye et al. (1998): Mali. Raymond et al. (1984): Senegal. Dias (1987): Guinea, Guinea Bissau. Inaoka et al. (1988), Ahmed et al. (2005): Nigeria. Amoudi \& Leclercq (1988, 1992, 1996), Amoudi (1989), Leclercq (2000), AlDhafer et al.(2009): Saudi Arabia. Dirie et al. 1989): Somalia. Turnbull et al. (1992): Ethiopia. Amsler et al. (1994), Solano \& Amsler-Delafosse (1995), Desquesnes \& Dia (2003), Dia et al. (2004), Koné et al. (2011): 
Burkina Faso. Chainey \& Cheke (1994): Togo. Dias (1994): Angola. Taylor \& Chainey (1994), Acapovi et al. (2001): Ivory Coast. Dias (1996): CAR. Al-Houty (1997): Kuwait. Mihok (2002): Kenya. Schacht (2002): Gambia. Abdesalam et al. (2003): Chad. Esterhuizen (2006): Natal Province - South Africa. Müller et al. (2011): Jordan. Müller et al. (2012): Sinai Peninsula and Israel. Abu El-Hasan et al. (2010, 2013): Egypt. Abu El-Hasan et al. (2013): Mozambique. Baldacchino et al. (2014): Africa and Middle East. Bitome Essono et al. (2015): Gabon.

Distribution (only verified precise location from the last period): Africa and Middle East, Aldabra Island, Angola, Burkina Faso, Cape Province - Natal, Cameroon, CAR, Chad, Congo Republic, DRC, Egypt, Eritraea, Ethiopia, Gabon, Gambia, Guinea, Guinea Bissau, Israel, Ivory Coast, Kenya, Kuwait, Madagascar, Mali, Mozambique, Natal Province - South Africa, Nigeria, Rhodesia, Saudi Arabia, Senegal, Sinai Peninsula, Somalia, Sudan, Tanzania, Togo, Uganda.

18. Tabanus thoracinus Palisot de Beauvois, 1807: Ins. rec. Afr. Amer., p. 55.

Material examined: Gabon, Loango NP, Petit Loango, 3 우, 10.-17.v.2014, 1 22.-25.v.2014, MT, J. Brzoňová leg. (dry, pined, NMPC). Liberia, VIRO camp, Sapo NP, 5¹8'39.89"N, $8^{\circ} 46^{\prime} 53.07^{\prime \prime} \mathrm{W}, 130$ a.s.l., dense forest, 1 o, ix./x. 2016, MT, J. Votýpka leg.; same, Jelay x VIRO, 5²0'14.92"N, 847'58.44"W, 125 m a.s.1., river, forest, 2 + $\circ$, ix./x. 2016, MT, J. Votýpka leg., (dry, pined, NMPC).

Chronology of selected important zoogeographical views. Oldroyd (1954): from Sierra Leone to former Portuguese East Africa, and across to Angola (adapted). Leclercq (1961): former Congo (adapted). Moucha (1976): Ethiop. Region. Chainey \& Oldroyd (1980): Nigeria; throughout Afrotrop. Reg. (excl. Malagasian subregion), Ivory Coast. Dusbábek et al. (1980): Uganda. Goodwin (1982): Mali. Raymond et al. (1984): Senegal. Dias (1987): Lunda Tchokwe (adapted). Hayakawa et al. (1989): Nigeria. Dias (1994): Angola. Dias (1996): CAR. Sasaki \& Nishida (1999), Sasaki (2005): Tanzania, Mihok (2002): Kenya.

Distribution (only verified precise location from the last period): Angola, CAR, Congo Republic, DRC, Ivory Coast, Kenya, Mali, Nigeria, Senegal, Sierra Leone Republic, Tanzania, Uganda. Published as new for Gabon and Liberia by Votýpka et al. (2019).

19. Tabanus triquetrornatus Carter, 1915: Ann. Trop. med. Paras., 9: 173.

Material examined: CAR, Dzanga-Sangha NP, Bai Hokou, Makumba, nest, 1 + , 26.ix.2012, J. Votýpka leg. (dry, pined, NMPC).

Chronology of selected important zoogeographical views. Oldroyd (1954): Nigeria, Sierra Leone, former Belgian Congo, British and French Cameroons (adapted). Moucha (1976): W. and C. Africa. Chainey \& Oldroyd (1980): Nigeria; Angola, Ivory Coast, Liberia, Sierra Leone, former Zaire, Cameroun and Congo (adapted). Inaoka et al. (1988): Nigeria. Dias (1994): Angola. Taylor \& Chainey (1994): Ivory Coast.

Distribution (only verified precise location from the last period): Angola, Cameroon, Congo Republic, DRC, Ivory Coast, Liberia, Nigeria, Sierra Leone Republic. Published as new for CAR by Votýpka et al. (2019).

20. Tabanus variabilis Loew, 1858: Öfv. Kongl. Vet.-Akad. Forh., 14: 340.

Material examined: CAR, Dzanga-Sangha NP, Bayanga, Bai Hokou, camp, 1 ㅇ, 26.ix.2012, MT, J. Votýpka leg.; same, Bai-Gubunga, 2 우, 28.ix.2012, MT, J. Votýpka leg. (dry, pined, NMPC).

Chronology of selected important zoogeographical views. Oldroyd (1954): from the former Cape Colony to Uganda, Kenya and the southern Sudan, westwards to Sierra Leone, Gambia and former French Equatorial Africa (adapted). Moucha (1976): S. and E. Africa. Chainey \& Oldroyd (1980): South Africa; widespread W. Afr. to Sudan, E. Afr. and southern Afr., ? former Congo (adapted). Raymond et al. (1984): Senegal. Dias (1994): Angola. Dias (1996): CAR. Esterhuizen (2006): Natal Province - South Africa.

Distribution (only verified precise location from the last period): Angola, CAR, Congo Republic, DRC, Gambia, Kenya, Natal Province - South Africa, Senegal, Sierra Leone Republic, Sudan, Uganda.

\section{HAEMATOPOTINI}

Haematopota Meigen, 1803: Illig. Mag. Ins., 2: 267.

21. Haematopota albihirta Karsch, 1887: Berl. ent. Zeit., 31: 371.

Material examined: Gabon, Loango NP, Petit Loango, 1 \&, 22.-25.v.2014, MT, J. Brzoňová leg. (dry, pined, NMPC). 
Chronology of selected important zoogeographical views. Oldroyd (1952): Somaliland, Abyssinia, Sudan, Uganda, Kenya, Tanganyika, Transvaal, Natal, former Zululand, Basutoland, S. Rhodesia, Nyasaland, Port. E. Africa, Belgian Congo and Portuguese Guinea (adapted). Leclercq (1961): former Congo (adapted). Moucha (1976): E., C. and S. Africa. Chainey \& Oldroyd (1980): Tanzania; Angola, Ethiopia, Kenya, Lesotho, Malawi, Mozambique, Rhodesia, Somalia, South Africa, Sudan, Uganda, Zambia, former Zaire (adapted). Phelps \& Holloway (1990): Zimbabwe. Turnbull et al.(1992): Ethiopia. Dias (1994): Angola. Sasaki (2005): Tanzania.

Distribution (only verified precise location from the last period): Angola, Congo Republic, DRC, Ethiopia, Guinea Bissau, Kenya, Lesotho, Malawi, Mozambique, Rhodesia, Somalia, South Africa, Sudan, Tanzania, Uganda, Zambia, Zimbabwe. Published as new for Gabon by Votýpka et al. (2019).

22. Haematopota bowdeni Oldroyd, 1952: Horse-flies Ethiop. Reg., 1: 95.

Material examined: Gabon, Loango NP, Akaka, 1 ㅇ, 28.vi.-9.vii.2014, MT, J. Brzoňová leg. (dry, pined, NMPC).

Chronology of selected important zoogeographical views. Oldroyd (1952): Liberia, former Gold Coast (adapted). Leclercq (1965): Liberia. Moucha (1976): Ghana, Liberia. Chainey \& Oldroyd (1980): Ghana, Liberia. Taylor \& Chainey (1994): Ivory Coast.

Distribution (only verified precise location from the last period): Ghana, Ivory Coast, Liberia. Published as new for Gabon by Votýpka et al. (2019).

23. Haematopota brucei Austen, 1908: Ann. Mag. nat. Hist., (8) 1: 413.

Material examined: CAR, Dzanga-Sangha NP, Bai Hokou, Makumba, 1 †, 26.ix.2012, nest (SW ?), J. Votýpka leg.; Gabon, Loango NP, Petit Loango, 1 क, 10.-17.v.2014, MT, J. Brzoňová leg. (dry, pined, NMPC).

Chronology of selected important zoogeographical views. Oldroyd (1952): Uganda, S. Sudan, former Belgian Congo (adapted). Moucha (1976): Sudan, Uganda, former Congo (adapted). Chainey \& Oldroyd (1980): Uganda; Ivory Coast, Sudan, former Zaire (adapted). Dias (1996): CAR.

Distribution (only verified precise location from the last period): CAR, Congo Republic, DRC, Ivory Coast, Sudan, Uganda. Published as new for Gabon by Votýpka et al. (2019).

24. Haematopota ciliatipes Bequaert, 1930: Harvard-Afr. Exp. Liberia, 36: 956.

Material examined: CAR, Dzanga-Sangha NP, Bai-Gubunga, 2 q $q$, 28.ix.2012, MT, J. Votýpka leg. (dry, pined, NMPC).

Chronology of selected important zoogeographical views. Oldroyd (1952): Cameroon, former Belgian Congo, Sudan (adapted). Moucha (1976): Sudan, former Congo, Cameroon (as ciliatipennis, adapted). Chainey \& Oldroyd (1980): former Zaire; Cameroon, Sudan (adapted). Dias (1996): CAR.

Distribution (only verified precise location from the last period): Cameroon, CAR, Congo Republic, DRC, Sudan.

25. Haematopota furians Edwards, 1916: Bull. ent. Res., 7: 157.

Material examined: Liberia, Jelay town (Jatia town), $5^{\circ} 21^{\prime} 11.23^{\prime \prime} \mathrm{N}, 8^{\circ} 48^{\prime} 39.59^{\prime \prime} \mathrm{W}, 120$ a.s.1., village, secondary forest, 1 q, ix./x. 2016, MT, J. Votýpka leg.; same, VIRO camp, Sapo NP, 5¹8'39.89"N, 846'53.07"W, 130 a.s.l., dense forest, 2 우, ix./x. 2016, MT, J. Votýpka leg. (all dry, pined, NMPC).

Chronology of selected important zoogeographical views. Oldroyd (1952): Sierra Leone, Liberia, Senegal, former French Guinea (adapted). Moucha (1976): W. Africa. Chainey \& Oldroyd (1980): Sierra Leone, Guinea, Guinea-Bissau, Liberia, Mali, Senegal. Raymond et al. (1984): Senegal.

Distribution (only verified precise location from the last period): Guinea, Guinea Bissau, Liberia, Mali, Senegal, Sierra Leone Republic.

26. Haematopota griseicoxa Oldroyd, 1952: Horse-flies Ethiop. Reg., 1: 52.

Material examined: CAR, Dzanga-Sangha NP, Bai-Gubunga, 2 우, 28.ix.2012, MT, J. Votýpka leg.; same, Bai Hokou, Makumba, nest, 1 , 26.ix.2012, J. Votýpka leg. (all dry, pined, NMPC).

Chronology of selected important zoogeographical views. Oldroyd (1952): S. Sudan, former Belgian Congo and British Cameroons (adapted), Moucha (1976): C. Africa. Chainey \& Oldroyd (1980): Cameroon; Ivory Coast, Nigeria, Sudan, former Congo and Zaire (adapted). Dias (1996): CAR.

Distribution (only verified precise location from the last period): Cameroon, CAR, Congo Republic, DRC, Ivory Coast, Nigeria, Sudan. 
27. Haematopota guineensis Bigot, 1891: Bull. Soc. zool. France, 16: 76.

Material examined: Liberia, VIRO camp, Sapo NP, 5¹8'39.89"N, 846'53.07"W, 130 a.s.1., dense forest, 1 , ix./x. 2016, MT, J. Votýpka leg., (dry, pined, NMPC).

Chronology of selected important zoogeographical views. Oldroyd (1952): Angola, Ivory Coast, S. Nigeria, Uganda, former Gold Coast, Cameroons, French Congo, Belgian Congo (adapted). Leclercq (1965): Liberia. Moucha (1976): Ethiop. Region. Chainey \& Oldroyd (1980): Ivory Coast, Angola, Cameroon, Central African Republic, Ghana, Guinea, Liberia, Nigeria, Uganda, former Congo and Zaire (adapted). Hayakawa et al. (1989): Nigeria. Dias (1994): Angola. Dias (1996): CAR.

Distribution (only verified precise location from the last period): Angola, CAR, Cameroon, Congo Republic, DRC, Ghana, Guinea, Ivory Coast, Liberia, Nigeria, Uganda.

28. Haematopota torquens Austen, 1908: Ann. Mag. Nat. Hist. 1(8): 409.

Material examined: Liberia, VIRO camp, Sapo NP, 5¹8'39.89"N, $8^{\circ} 46^{\prime} 53.07^{\prime \prime} \mathrm{W}, 130$ a.s.1., dense forest, 1 , ix./x. 2016, MT, J. Votýpka leg., (dry, pined, NMPC).

Chronology of selected important zoogeographical views. Oldroyd (1952): Sierra Leone, Liberia, former Gold Coast (adapted). Moucha (1976): Sierra Leone, Liberia, Ghana. Chainey \& Oldroyd (1980): Ghana, Angola, Ivory Coast, Liberia, Sierra Leone, former Zaire (adapted). Dias (1994): Angola. Taylor \& Chainey (1994): Ivory Coast.

Distribution (only verified precise location from the last period): Angola, DRC, Ghana, Ivory Coast, Liberia, Sierra Leone Republic.

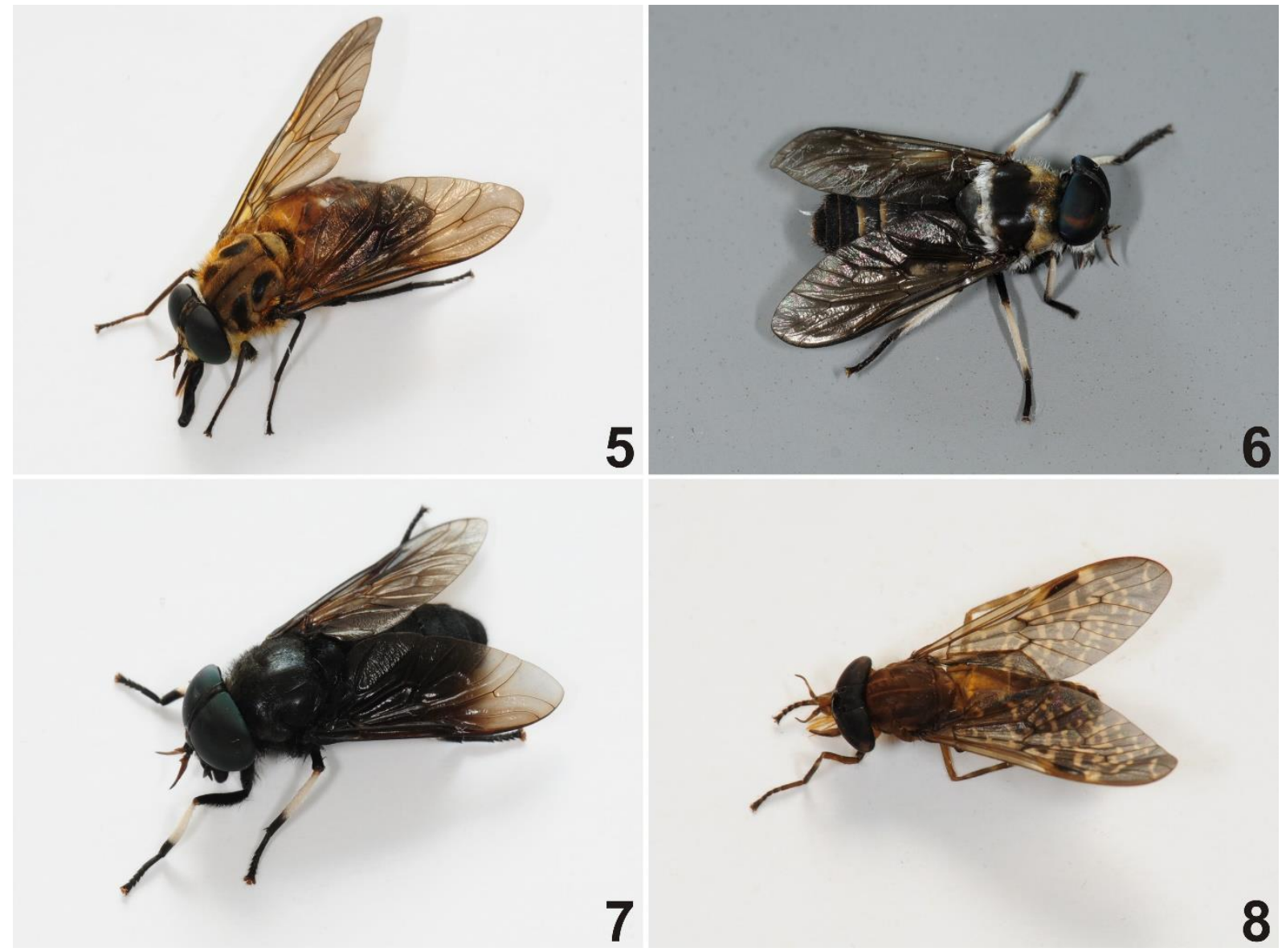

Figs 5-8: Documentary photos of: 5 - Philoliche (Subpangonia) gravoti Surcouf, 1908 from Liberia, 6 - Tabanus argenteus Surcouf, 1907 from Liberia, 7 - Tabanus boueti Surcouf, 1907 from Liberia, 8 - Haematopota furians Edwards, 1916 from Liberia (all photos made by D. Modrý). 
Acknowledgements: Our thanks are due to Aleš Bezděk (Czech Academy of Sciences, České Budějovice, CZ) and Michal Tkoč (Department of Entomology, National Museum, Praha, CZ) for copies of many published papers from around the world and information on new literature, and also David Modrý (Department of Pathological Morphology and Parasitology, University of Veterinary and Pharmaceutical Sciences Brno, Brno, CZ) for permission to use his photograph of family Tabanidae from Liberia. We would especially like to thank to anonymous reviewers, whose helped by providing constructive comments, as well as for improving the manuscript. The work was partially supported by the Ministry of Culture of the Czech Republic (DKRVO 2019-2023/5.I.a, National Museum, 00023272) and by the ERD Funds, project CePaViP (CZ.02.1.01/0.0/0.0/16_019/0000759).

\section{References}

Abdesalam A.D., Delafosse A., Elsen P. \& Amsler-Delafosse S. (2003): Vecteurs potentiels de Trypanosoma evansi chez le dromadaire au Tchad oriental. - Révue d' Élevage et de Médecine vétérinaire des Pays tropicaux 55(1) /2002/: 2130 .

Abu El-Hassan G.M.M., Badrawy H.B.M., Mohammad S.K. \& Fadl H.H. (2010): Cladistic analysis of Egyptian horse flies (Diptera: Tabanidae) based on morphological data. - Egyptian Academic Journal of Biological Sciences 3(2): 51-62.

Abu El-Hassan G.M.M., Badrawy H.B.M. \& Fadl H.H. (2013): A review of the genus Tabanus Linnaeus, 1758 (Diptera: Tabanidae) from Egypt. - Zootaxa 3691(5): 559-576.

Acapovi G.L., Yao, Y., N'Goran E., Dia M.L. \& Desquesnes M. (2001): Abondance relative des tabanidés dans la région des savanes de Côte d'Ivoire. - Revue d'Élevage et de Médecine vétérinaire Pays tropicaux 54(2): 109-114.

Ahmed A.B., Okiwelu S.N. \& Samdi S.M. (2005): Species diversity, abundance and seasonal occurrence of some biting flies in Southern Kaduna, Nigeria. - African Journal of Biomedical Research 8: 113-118.

A1-Dhafer H.M., Dawah H.A. \& Abdullah M.A. (2009): Tabanidae (Diptera) of Saudi Arabia. - Saudi Journal of Biological Sciences 16: 77-83.

A1-Houty W. (1997): Checklist of the insect fauna of Kuwait. - Kuwait Journal of Science \& Engineering 24(1): 145-162.

Amoudi M.A. (1989): New records of Tabanidae (Diptera) from southwest Saudi Arabia with some aspects on their descriptions and biological information. - Journal of Biological Sciences Research 20(1): 115-127.

Amoudi M.A. \& Leclercq M. (1988): Tabanus riyadhae (Diptera: Tabanidae), a new species from Saudi Arabia. - Journal of Medical Entomology 25(5): 399-401.

- (1992): The horse-flies from Saudi Arabia, distribution and zoogeography (Diptera: Tabanidae). - Notes fauniques de Gembloux 25: 3-15.

- (1996): New records of Tabanidae (Diptera) from Saudi Arabia, first record of Atylotus venturii Leclercq and Tabanus separatus Efflatoun. - Journal of the Egyptian Society of Parasitology 26(1): 1-7.

Amsler S., Filledier J. \& Millogo R. (1994): Attractivité pour les Tabanidae de différents pièges à glossines avec ou sans attractifs olfactifs. Résultats préliminaires obtenus au Burkina Faso. - Revue d'Élevage et de Médecine véterinaire Pays tropicaux 47(1): 63-68.

Baldacchino F., Desquesnes M., Mihok S., Foil L.D., Duvallet G. \& Jittapalapong S. (2014): Tabanids: Neglected subjects of research, but important vectors of disease agents! - Infection, Genetics and Evolution 28: 596-615.

Bitome Essono P.Y., Dechaume-Moncharmont F.-X., Mavoungou J., Mba R.O., Duvallet G. \& Bretagnolle F. (2015): Distribution and abundance of hematophagous flies (Glossinidae, Stomoxys, and Tabanidae) in two national parks of Gabon. - Parasite 22(23): 1-11.

Caubère P. \& Noireau F. (1991): Effect of attraction factors on the sampling of Chrysops silacea and C. dimidiata (Diptera: Tabanidae), vectors of Loa loa (Filaroidea: Onchocercidae) filariasis. - Journal of Medical Entomology 28(2): 263-265.

Caubère P., Noireau F. \& Frezil J.-L. (1990): Mise en évidence de Trypanosomes de reptiles chez Chrysops silacea et C. dimidiata (Diptera: Tabanidae) au Sud-Congo. - Annales de Parasitologie Humaine et Comparee 65(3): 149.

Chainey J. (2017): 39. Tabanidae (Horse Flies, Deer Flies and Clegs). In: Kirk-Spriggs A.H. \& Sinclair B.J. (eds): Manual of Afrotropical Diptera. Volume 2. Nematocerous Diptera and lower Brachycera. Suricata, 5. South African National Biodiversity Institute, Pretoria, pp. 893-913.

Chain ey J.E. \& Cheke R.A. (1994): A collection of Tabanidae (Diptera) from Togo with two new synonyms in Haematopota. - Journal of African Zoology 108: 225-229.

Chain ey J.E. \& Oldroyd H. (1980): Family Tabanidae. pp. 275-308. In: Crosskey R.W. (ed.), Catalogue of the Diptera of the Afrotropical Region. British Museum (Natural History), London, $1437 \mathrm{pp}$.

Cheke R.A., Mas J. \& Chainey J.E. (2003): Potential vectors of loiasis and other tabanids on the island of Bioko, Equatorial Guinea. - Medical and Veterinary Entomology 17: 221-223.

Chippaux J.-P., Bouchité B., Demanou M., Morlais I. \& Le Goff G. (2000): Density and dispersal of the loaiasis vector Chrysops dimidiata in southern Cameroon. - Medical and Veterinary Entomology 14: 339-344.

Demanou M., Pion S.D.S. \& Boussinesq M. (2001): Étude entomologique sur la transmission de la loase dans le département de la Lékie (Cameroun). - Bulletin de la Société de pathologie exotique 94(4): 347-352.

Desquesnes M. \& Dia M.L. (2003): Trypanosoma vivax: mechanical transmission in cattle by one of the most common African tabanids, Atylotus agrestis. - Experimental Parasitology 103: 35-43.

Dia M.L., Desquesnes M., Elsen P., Lancelot R. \& Acapovi G. (2004): Evaluation of a new trap for Tabanids and Stomoxyines. - Bulletin Societé royale belge d'Entomologie 140: 72-81.

Dias J.A.T.S. (1985): Tabanídeos (Diptera-Tabanidae) recolhidos pela missão zoologica da „SPEN“ a republica da GuinéBissau. - Boletim da Sociedade Portuguesa de Entomologia 71: 1-5.

- (1987): Tabanídeos (Diptera-Tabanidae) provenientes de alguns antigos territórios portugueses de África, em colecção no Centro de Zoologia. - Garcia de Orta, Sér. Zool., Lisboa, 14(1): 89-101. 
- (1992): Uma nova espécie de tabanídeo (Diptera, Tabanidae), da Guiné-Bissau Tabanus mesquitelai n. sp. - Garcia de Orta, Sér. Zool., Lisboa 17(1-2) (1990): 27-30.

- (1994): Descoberta de uma nova espécie do género Tabanus Linnaeus, 1758 (Diptera - Tabanidae) para a fauna de Angola. - Garcia de Orta, Sér. Zool., Lisboa 20(1-2): 69-76.

- (1996): Contribuição para o conhecimento dos Tabanídeos (Diptera-Tabanidae) da República Centro-Africana. - Garcia de Orta, Sér. Zool., Lisboa 21(1): 67-80.

Dirie M.F., Wallbanks K.R., Aden A.A., Bornstein S. \& Ibrahim M.D. (1989): Camel Trypanosomiasis and its vectors in Somalia. - Veterinary Parasitology 32: 285-291.

Djiteye A., Diarra M., Ouattara I. \& Traore D. (1998): Comparison of the efficacy of different traps and attractans for Tabanidae and Stomoxys in Mali. - Journal of Protozoology Research 8: 263-273.

Dusbábek F., Gregor F. \& Daniel M. (1980): Note on some Diptera (Tabanidae, Glossinidae, Muscidae and related families) at the peak of dry season in Toro Game Reserve, Uganda. - Folia parasitologica, Praha 27: 165-171.

Esterhuizen J. (2006): Seasonal abundance of horse flies (Diptera: Tabanidae) from two conservation areas in northeastern KwaZulu-Natal Province, South Africa. - African Entomology 14(2): 395-397.

Goodwin J.T. (1982): The Tabanidae (Diptera) of Mali. - Miscellaneous Publications of the Entomological Society of America 13(1): 1-141.

- (1985): Notes on the seasonal and spatial distribution of Tabanidae (Diptera) at Forệt Classee de Tienfala, Republic of Mali. - Myia 3: 415-431.

Gouteux J.P., Noireau F. \& Staak C. (1989): The host preferences of Chrysops silacea and C. dimidiata (Diptera: Tabanidae) in an endemic area of Loa loa in the Congo. - Annals of Tropical Medicine and Parasitology 83(2): 167-172.

Hayakawa H., Watanabe M. \& Takahasi H. (1989): Surveys of tabanid flies in the early stage of the dry season in Nigeria. - Japanese Journal of Sanitary Zoology 40(2): 117-122.

Iboh C.I., Okon O.E., Arong G.A., Asor J.E. \& Opara K.N. (2012): Occurrence and distribution of Chrysops species in Akamkpa Community of Cross River State, Nigeria. - Pakistan Journal of Biological Sciences 15(23): 1139-1143.

Itina V.I., Noutcha A.M.E. \& Okiwelu S.N. (2013): Spatial and temporal distribution of Tabanids (Diptera: Tabanidae) in Akwa Ibom State, Nigeria. - Research in Zoology 3(2): 62-65.

Inaoka T., Hori E., Yamaguchi K., Watanabe M., Yoneyama Y. \& Ogunba E.O. (1988): Morphology and identification of Chrysops larvae from Nigeria. - Medical and Veterinary Entomology 2: 141-152.

Inaoka T., Hori E., Ogunba E.O., Watanabe M., Yamaguchi K., Yoneyama Y., Amano T., Takaoka M. \& Maeda R. (1988): Morphological characteristics of the anal segment in the identification of the larvae of Tabanus-species (Diptera, Tabanidae) collected from Nigeria. - Japanese Journal of Sanitary Zoology 39(3): 277-282.

Karim A.E.I. (1980): Studies on the life-histories of some Tabanidae of Southern Darfur, Sudan. (1) Seasonal and daily abundance. - The Sudan journal of veterinary science and animal husbandry 21(2): 66-76.

Kelly-Hope L.A., Bockarie M.J. \& Molyneux D.H. 2012: Loa loa ecology in Central Africa: Role of the Congo River System. - PLOS neglected tropical diseases 6(6): 1-4.

Koné N., N'Goran E.K., Sidibe I., Kombassere A.W. \& Bouyer J. (2011): Spatio-temporal distribution of tsetse and other biting flies in the Mouhoun River basin, Burkina Faso. - Medical and Veterinary Entomology 25: 156-168.

Leclercq M. (1961): Tabanidae (Diptera Brachcera). - Parc National de la Garamba, Mission H. De Saeger 21(5): 99-116.

- (1965): Tabanidae (Diptera) du Liberia et d'autres pays Africains. - Extrait du Bulletin de 1'Institut Agronomique et des Stations de Recherches de Gembloux 33(1): 135-137.

- (2000): A faunistic account of Tabanidae (Diptera) of Saudi Arabia and Oman. - Fauna of Arabia 18: 285-292.

Mahmoud M.M. \& Osman M.O. (1979): A note on trypanosomiasis in Sudan camels, Scandinavian Institute of African Studies, Uppsala, pp. 502-508.

Mavoungou J.F., Makanga B.K., Acapovi-Yao G., Desquesnes M. \& M'Batchi B. (2012): Chorologie des Tabanidae (Diptera) dans la réserve de biosphère Ipassa-Makokou (Gabon) en saison des pluies. - Parasite 19: 165-171.

Mihok S. (2002): The development of a multipurpose trap (the Nzi) for tsetse and other biting flies. - Bulletin of Entomological Research 92: 385-403.

Mohamed H.I., Hassan M.I., Fouda M.A. \& Bahgat D.F. (2002): Ultrastructure of the mouth parts and associated sensilla of Tabanus taeniola and Haematopota sp. (Diptera: Tabanidae). - Journal of the Egyptian German Society of Zoology 39E: 91-106.

Morita S.I. (2008): A phylogeny of long-tongued horse flies (Diptera: Tabanidae: Philoliche) with the first cladistic review of higher relationships within the family. - Invertebrate Systematics 22: 311-327.

Morsy T.A. \& Habib K.S.M. (2001): Two species of Tabanids (order: Diptera) in Aswan district, Egypt. - Journal of the Egyptian Society of Parasitology 31(2): 429-432.

Mou cha J. (1976): Horse-flies (Diptera, Tabanidae) of the World. Synoptic Catalogue. - Acta Entomologica Musei Nationalis Pragae, suppl. 7: 1-319.

Müller G.C., Hogsette J.A., Revay E.E., Kravchenko V.D., Leshvanov A. \& Schlein Y. (2011): New records for the horse fly fauna (Diptera: Tabanidae) of Jordan with remarks on ecology and zoogeography. - Journal of Vector Ecology 36(2): 447-450.

Müller G.C., Revay E.E., Hogsette J.A., Zeegers T., Kline D., Kravchenko V.D. \& Schlein Y. (2012): An annotated checklist of the horse flies (Diptera: Tabanidae) of the Sinai Peninsula Egypt with remarks on ecology and zoogeography. - Acta Tropica 122: 205-211.

Noireau F. \& Gouteux J.P. (1989): Current considerations on a Loa loa simian reservoir in the Congo. - Acta Tropica 46: $69-70$.

Noireau F., Nzoulani A., Sinda D. \& Caubère P. (1991): Chrysops silacea and C. dimidiata seasonality and loiasis prevalence in the Chaillu mountains, Congo. - Medical and Veterinary Entomology 5: 413-419. 
Noireau F., Nzoulani A., Sinda D. \& Itoua A. (1990a): Chrysops silacea and C. dimidiata: fly densities and infection rates with Loa loa in the Chaillu mountains, Congo Republic. - Transactions of the Royal Society of Tropical Medicine and Hygiene 84: 153-155.

- (1990b): Transmission indices of Loa loa in the Chaillu Mountains, Congo. - American Journal of Tropical Medicine and Hygiene 43(3): 282-288.

Oldroyd H. (1952): The horse-flies (Diptera, Tabanidae) of the Ethiopian region. Vol. I: Haematopota and Hippocentrum. British Museum (Natural History), London, $226 \mathrm{pp}$.

- (1954): The horse-flies (Diptera, Tabanidae) of the Ethiopian region. Vol. II: Tabanus and related genera. British Museum (Natural History), London, $341 \mathrm{pp}$.

- (1957): The horse-flies (Diptera, Tabanidae) of the Ethiopian region. Vol. III: Subfamilies Chrysopinae, Scepsidinae and Pangoniinae and a revised classification. British Museum (Natural History), London, $489 \mathrm{pp}$.

Phelps R.J. \& Holloway M.T.P. (1990): Alighting sites of female Tabanidae (Diptera) at Rekomitjie, Zimbabwe. - Medical and Veterinary Entomology 4: 349-356.

Pinder M. (1991): The improvement of maintenance conditions for wild-caught Chrysops silacea and the production of infective larvae of Loa loa. - Acta Tropica 49: 305-311.

Raymond H.L., Taufflieb R., Cornet M., Camicas J.-L., Chateau R. \& Dieng P.Y. (1984): Liste annotée des Tabanidae (Diptera) du Sénégal et de la Gambie. - Bulletin de l'Institut fondamental d'Afrique noire 42(4) (1980): 812822.

Sas aki H. (2005): Tabanid flies (Diptera: Tabanidae) of the Mahale Mountains National Park, Tanzania, East Africa. - Journal of Rakuno Gakuen University 30(1): 93-98.

Sasaki H. \& Nishida T. (1999): Notes on the flies associated with wild chimpanzees at Mahale Mountains National Park, Tanzania, East Africa. - Medical Entomology and Zoology 50(2): 151-155.

Schacht W. (2000): Insekten aus Gambia, Westafrika (Diptera: Platypezidae, Odiniidae, Tabanidae, Glossinidae und Planipennia: Chrysopidae, Myrmeleontidae, Ascalaphidae sowie Coleoptera: Carabidae, Cicindelidae, Elateridae, Scarabaeidae). - Entomofauna 21(1): 1-4.

- (2002): Weitere Insekten aus Gambia, Westafrika (Coleoptera: Buprestidae, Scarabaeidae; Diptera: Asilidae, Asteiidae, Tabanidae; Hymenoptera: Apidae, Scoliidae; Planipennia: Ascalaphidae, Myrmeleontidae). - Entomofauna 23(13): 149154.

Solano P. \& Amsler-Delafosse S. (1995): Trypanosoma congolense chez différentes de taons (Diptera: Tabanidae) au Burkina Faso. - Revue d'élevage et de médecine vétérinaire des pays tropicaux 48(2): 145-146.

Steyskal G.C. \& El-Bialy S. (1967): A list of Egyptian Diptera with a bibliography and key to families. - Technical Bulletin, United Arab Republic, Ministry of Agriculture 3: 1-87.

Taylor P.D. \& Chainey J.E. (1994): Tabanidae (Diptera) from Taï National Park, Côte d' Ivoire with descriptions of three new species. Journal of African Zoology 108: 467-479.

Turnbull D.A., Taylor P.D., Smith S.M. \& Chainey J.E. (1992): A collection of Tabanidae (Diptera) from west-central Ethiopia, with descriptions of Tabanus gibensis sp. n. and the male of T. pallidifacies Surcouf. - Journal of African Zoology, 106: 133-140.

Votýpka J., Brzoňová J., Ježek J. \& Modrý D. (2019): Horse flies (Diptera: Tabanidae) of three West African countries: faunistic update, barcoding analysis and trypanosome occurrence. - Acta Tropica 197: 105069.

Wahl G., Moukagni R., Toure F. \& Georges A.J. (1995): Large scale collection of viable infective larvae of Loa loa. Tropical Medicine and Parasitology 46: 203-204.

Wanji S., Tendong for N., Esum M.E. \& Enyong P. (2002): Chrysops silacea biting densities and transmission potential in an endemic area of human loiasis in south-west Cameroon. - Tropical Medicine and International Health 7(4): 371-377.

Wiesenhütter E. (1980): Research into the relative importance of Tabanidae (Diptera) in mechanical disease transmission. III. The epidemiology of anaplasmosis in a Dar-es-Salaam dairy farm. - Tropical Animal Health and Production $7(1)(1975): 15-22$.

Authors’address: Jan Ježek, National Museum, Department of Entomology, Cirkusová 1740, CZ-193 00 Praha 9-Horní Počernice, Czech Republic E-mail: jan.jezek@o2active.cz

Jan Votýpka \& Jana Brzoňová, Charles University, Faculty of Science, Department of Parasitology, Viničná 7, CZ-128 44 Praha 1, Czech Republic E-mails: jan.votypka@natur.cuni.cz; jana.radrova@natur.cuni.cz

Jan Votýpka, Institute of Parasitology, Czech Academy of Sciences, České Budějovice, Czech Republic

Jozef Oboňa, University of Prešov, Faculty of Humanities and Natural Sciences, Department of Ecology, 17. novembra 1, SK-081 16 Prešov, Slovakia

${ }^{*}$ Corresponding author, e-mail: obonaj@centrum.sk 